\title{
First Auto-Magnifier Platform for Hardware Assurance and Reverse Engineering Integrated Circuits
}

\author{
Ronald Wilson $^{1 *}$, Navid Asadizanjani ${ }^{1}$, Domenic Forte ${ }^{1}$ and Damon L. Woodard ${ }^{1}$ \\ 1. Department of Electrical and Computer Engineering, University of Florida, Gainesville, USA \\ * Corresponding author: ronaldwilson@ufl.edu
}

Scanning Electron Microscopy (SEM) is a common imaging modality used in the semiconductor industry particularly for failure analysis $[1,2]$. In addition, with the recent growing concern of physical attacks on electronics with malicious intent, SEMs are becoming more attractive for hardware assurance and especially, Reverse Engineering (RE) [4]. However, RE requires long hours of imaging for an IC even if it is done automatically. In [3], the estimated time to image an IC of size $1.5 \mathrm{~mm} \times 1.5 \mathrm{~mm}$ employing a $130 \mathrm{~nm}$ node technology with high resolution went up to 30 days. With the ongoing trend of adding more structures onto a limited space on the IC, the imaging time frame is becoming unfeasible. This drawback would be manageable in situations where there is a prior knowledge on the exact location to be imaged. However, in case of hardware assurance and reverse engineering, where all the structures in the entire IC need to be extracted precisely without any prior knowledge, the drawback would be a major limiting factor. There are several parameters in the SEM imaging modality such as magnification that can be adjusted to reduce the time requirements. In [4], Principe et al. even introduced a platform for automated processing for an IC. However, both approaches do not consider the density of the structures being imaged and fails to investigate if the region warrants a high definition setting to resolve the features satisfactorily. In this paper, we introduce a new algorithm that can quantify the density of objects in a region using a fast-acquired low magnification image and define the right value of magnification for imaging different regions automatically. Once the density of the objects is quantified the microscope can increase the magnification with a predefined step $(10 \mathrm{X}, 100 \mathrm{X}, 1000 \mathrm{X}$, etc.) and collect new images but only from the region detected as a dense region. This loop will be repeated until the whole area is imaged with the right magnification. The images are assumed to be analyzed using advanced image analysis methods incorporated with machine learning and deep learning algorithms for hardware assurance. Therefore, as the image analysis algorithms are further developed a lower magnified image might be also acceptable for analysis. This will be used towards building an intelligent microscope that can considerably reduce the imaging time requirement which would greatly facilitate efficient hardware assurance and RE.

The SEM image in Fig. 1, shows a typical frame from an IC. The proposed method, firstly, requires the binarization of the image with a traditional image processing algorithm such as thresholding. It then involves the extraction of run-length encoded components in the image and the projection of the entire SEM image onto its axes. A run-length encoded component is defined as the length of sequence of adjacent pixels in the image with the same pixel value. The projection for an axis can be calculated as the sum of all run-length encoded components divided by the frequency of the corresponding component. The projections can also be normalized by the length of axes for easy visual interpretation. However, this normalization provides no additional benefit to the method. Intuitively, the method assigns a higher penalty for short run-length encoded components with high frequency and low penalty for long run-length encoded components with low frequency. This can be seen from Fig. 1. The projection values are then thresholded for a decision boundary. In the paper, the decision boundary is 
taken as the mean of the respective projection. However, depending on the type of material imaged, the decision boundary can be quantized to more than 2 regions based on the parameters in the imaging modality and time constraints on the image acquisition phase. Any region in the image having both projections less than the decision boundary are marked as high frequency and the rest as low frequency. Fig. 1 shows the result of the application of the method on the sample.

Comparing the results given in Fig. 1, the proposed method was able to localize high and low frequency regions of the image successfully. This information can then be used to dynamically change the imaging parameters, as necessary, for different regions of the sample rather than using a constant setting for the entire sample. Since low quality imaging takes significantly less time than high quality imaging [3], the time required to image a sample would only be constrained only by the high frequency regions present in the sample. We were able to reduce the imaging time by $20 \%$ for the $130 \mathrm{~nm}$ node technology IC shown in Fig. 1. In conclusion, depending on the complexity of the structures in the sample, application of our algorithm will cause a significant reduction in imaging time for the entire sample.

\section{References:}

[1] Soden, Jerry M., and Richard E. Anderson. "IC failure analysis: techniques and tools for quality reliability improvement." Proceedings of the IEEE 81.5 (1993): 703-715.

[2] May, Gary S., and Costas J. Spanos. Fundamentals of semiconductor manufacturing and process control. John Wiley \& Sons, 2006:95-96

[3] Vashistha, N., Rahman, M. T., Shen, H., Woodard, D. L., Asadizanjani, N., \& Tehranipoor, M. (2018). Detecting Hardware Trojans Inserted by Untrusted Foundry Using Physical Inspection and Advanced Image Processing. Journal of Hardware and Systems Security, 2(4), 333-344.

[4] Principe, E. L., Navid Asadizanjani, Domenic Forte, Mark Tehranipoor, Robert Chivas, Michael DiBattista, Scott Silverman, Mike Marsh, Nicolas Piche, and John Mastovich. "Steps Toward Automated Deprocessing of Integrated Circuits." ISTFA, 2017.
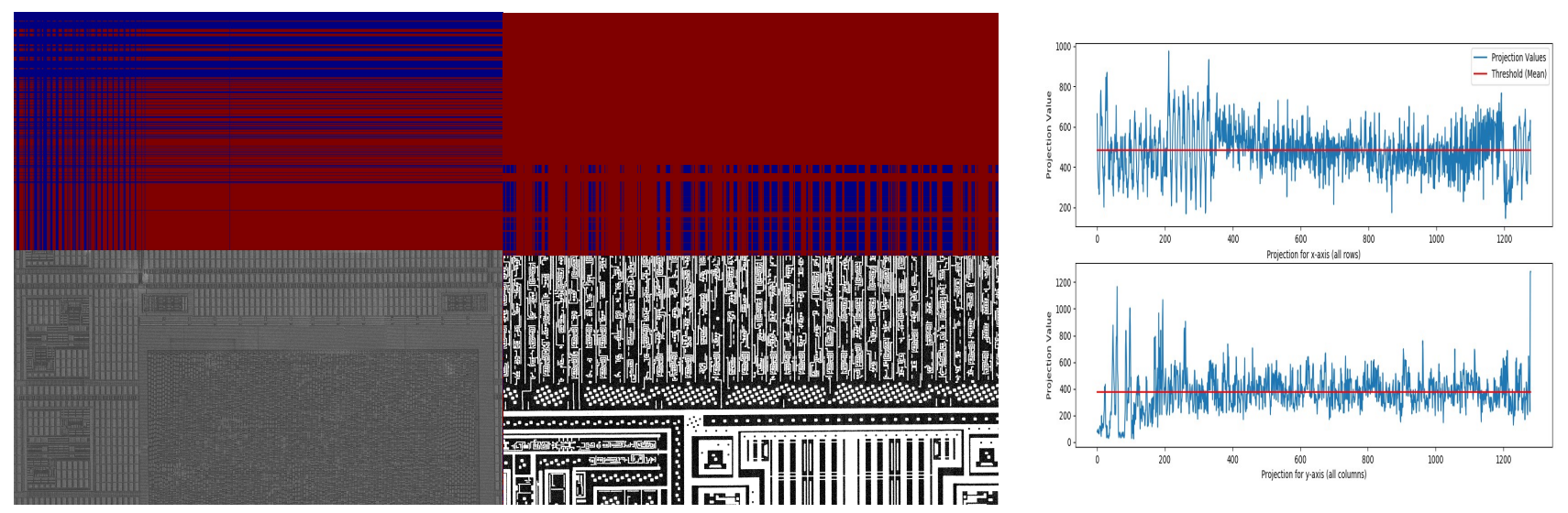

Figure 1. From the left, an SEM image of the active region of an IC employing 28nm node technology imaged at $200 \mu \mathrm{m}, 10 \mu \mathrm{s} /$ pixel, $1024 \times 1024$ at $5 \mathrm{kV}$ for the magnification, dwelling time, resolution and excitation voltage respectively. The center image shows a $130 \mathrm{~nm}$ IC with $100 \mu \mathrm{m}$ for magnification and rest of the parameters remaining the same. The colored region above the SEM image is the frequency map obtained from thresholding the row and column projections as shown in the image on the far right. Red shows high and blue shows low frequency. 